\title{
Comportamiento acústico y térmico de materiales basados en fibras naturales para la eficiencia energética en edificación
}

\section{Acoustic and thermal behavior of materials based on natural fibers for energy efficiency in buildings}

\author{
$\underline{\text { María Ángeles Navacerrada }}{ }^{(*)}$, Daniel de la Prida ${ }^{(*)}$, Alberto Sesmero ${ }^{(*)}$, Antonio Pedrero ${ }^{(*)}$, Tomás Gómez ${ }^{(* *)}$ y Patricia Fernández-Morales ${ }^{(* *)}$
}

\section{RESUMEN}

El uso de materiales aislantes es el primer paso para reducir la energía requerida para mantener una buena temperatura en el interior de un edificio y alcanzar la eficiencia energética. El objetivo es diseñar materiales para aislamiento acústico y térmico baratos, biodegradables y reciclables como los basados en fibras naturales. En este trabajo se estudian las propiedades térmicas y acústicas de no tejidos basados en fibras de fique, de coco y de algodón reciclado a partir de tejido denim. Se proponen posibles usos para los materiales fabricados basados en las exigencias del Código Técnico.

Palabras clave: fibras naturales; no tejidos; aislamiento térmico; aislamiento acústico.

\section{ABSTRACT}

Insulation materials are the first step towards energy efficiency and reducing energy consumption on buildings. The goal of this research is to design acoustic and thermal insulation materials which fulfill several criteria, low cost, biodegradable and recyclable, such as natural fiber-based materials. Thermal and acoustic properties of several nonwovens developed from fique, coir and recycled cotton from denim were characterized. Possible uses are proposed for the manufactured materials bases on the requirements of the Spanish Building Code.

Keywords: natural fibers; non woven; thermal isolation; acoustic isolation.

(*) Escuela Técnica Superior de Arquitectura, Universidad Politécnica de Madrid (España).

$\left.{ }^{* *}\right)$ Facultad de Ingeniería Industrial, Universidad Pontificia Bolivariana, Medellín (Colombia).

Persona de contacto/Corresponding author: mdelosangeles.navacerrada@upm.es (M. A. Navacerrada)

ORCID: https://orcid.org/oooo-ooo1-5142-1989 (M. A. Navacerrada); https://orcid.org/oooo-0002-4386-5544 (D. de la Prida); https://orcid.org/oooo-0oo1-5058-4903 (A. Sesmero); https://orcid.org/oooo-ooo1-9969-6308(A. Pedrero); https://orcid.org/ooooo001-5616-2699 (T. Gómez); https://orcid.org/oooo-0003-2344-1418 (P. Fernández-Morales)

Cómo citar este artículo/Citation: Navacerrada, María Ángeles; de la Prida, Daniel; Sesmero, Alberto; Pedrero, Antonio; Gómez, Tomás; Fernández-Morales, Patricia (2021). Comportamiento acústico y térmico de materiales basados en fibras naturales para la eficiencia energética en edificación. Informes de la Construcción, 73(561): e373. https://doi.org/10.3989/ic.74558

Copyright: (C) 2021 CSIC. Este es un artículo de acceso abierto distribuido bajo los términos de la licencia de uso y distribución Creative Commons Reconocimiento 4.0 Internacional (CC BY 4.0). 


\section{INTRODUCCIÓN}

La necesidad de confort en el interior de los recintos ha favorecido el desarrollo de técnicas y materiales para el aislamiento térmico y acústico. Además el aislamiento térmico influye en el plan general de la lucha contra el despilfarro energético. Las ideas de sostenibilidad, eficiencia energética y de respeto por el medio ambiente han calado en la sociedad y esto obliga en el sector de la construccion, entre otros, al desarrollo de nuevos materiales. En este punto, el papel social del arquitecto es de gran relevancia, sus decisiones para la selección e integración de los distintos materiales en el edificio pueden tener implicaciones importantes para el medio ambiente. El uso de materiales aislantes es el primer paso para reducir la energía requerida para mantener una buena temperatura en el interior y alcanzar la eficiencia energética. El mercado de los aislantes térmicos se encuentra en auge, debido en su mayor parte a estas exigencias y campañas a favor de los edificios de demanda energética nula. Los distintos fabricantes han supuesto el motor del cambio en el campo de la construcción. Cada fabricante intenta ofrecer nuevos productos con mejores prestaciones para dar solución a estas necesidades.

Los materiales aislantes térmicos se caracterizan por una baja conductividad térmica y por sus características algunos son empleados a la vez para el aislamiento térmico y acústico. Por ello, cada vez más el objetivo es diseñar materiales con un alto rendimiento acústico y térmico y que sean baratos, biodegradables y reciclables. En esta línea se encuentran los materiales basados en fibras naturales. Estos materiales no contienen plásticos ni ningún componente derivado del petróleo. Tampoco contienen sustancias o aditivos que puedan ser perjudiciales para la salud. Sin embargo tienen algunos inconvenientes como menor resistencia mecánica que las fibras sintéticas y alta absorción de humedad. La clasificación de las fibras está relacionada con la parte de la planta que se aprovecha: procedente del fruto y semillas, procedente del tallo y procedente de las hojas (1). Las fibras de origen vegetal están constituidas principalmente por celulosa, lignina, pectina y hemicelulosa y su contenido influye en sus propiedades (2). El contenido de celulosa influye en las propiedades macroscópicas de las fibras vegetales. La resistencia a la tracción y el módulo de Young aumentan con el contenido de celulosa (1). La lignina les proporciona mayor resistencia al ataque de los microorganismos (3) y la pectina flexibilidad a las fibras (4). Así mismo, un mayor contenido de hemicelulosa aumenta la absorción de humedad y acelera el proceso de biodegradación (5).

Las fibras naturales pueden tejerse para producir un tejido o apelmazarse para producir un no tejido. El no tejido está constituido por una red de fibras unidas por procedimientos mecánicos, térmicos o químicos pero sin ser tejidas y sin que sea necesario convertir las fibras en hilo. El resultado es una lámina de fibras flexibles y porosas sin trama (6). En este trabajo se han utilizado tres fibras naturales: el fique procedente de las hojas, la fibra de coco procedente del fruto y el algodón procedente de la semilla. En el caso del algodón las fibras resultan del deshilachado de tejido denim reciclado. Se han fabricado no tejidos por unido mecánico mediante la técnica del punzonado (6) a partir de estas fibras, todas ellas de igual densidad y espesor. Se han caracterizado acústica y térmicamente y se han analizado sus posibilidades de uso en construcción a partir de los parámetros de exigencia del Código Técnico de la Edificación (CTE) español en sus Documentos Básicos de Ahorro de Energía (DB-HE) y de Protección frente al Ruido (DB-HR). Se proponen posibilidades de uso de estos materiales y se analiza su coste de fabricación.

\section{MATERIALES}

En este apartado se describen las características de las fibras utilizadas y el método de fabricación de las muestras a partir de las fibras.

\subsection{Fibras naturales y recicladas}

A continuación se describen brevemente las características y propiedades de las fibras utilizadas:

Fique. Su nombre botánico es Furcraea Macrophylla, también conocida con el nombre de sisal. Es una de las fibras vegetales más representativas de la América tropical. Presenta unas hojas lanceoladas, de entre medio metro y dos metros de largas y entre 8 y $15 \mathrm{~cm}$ de anchura de cuyo interior se obtiene una fibra dura y fina, duradera, relativamente flexible y resistente al agua salada (7). Su diámetro oscila entre los 0,15 y 0,30 mm. El fique puede soportar hasta aproximadamente los $220^{\circ} \mathrm{C}$ sin degradarse. No absorbe la humedad fácilmente, su absorción del agua es del 60\% (8). Sus principales aplicaciones son la cordelería y los empaques. En la fabricación de las muestras se usaron los desechos que resultan de la fibra larga de fique usada para la fabricación de empaques. El uso adicional de este deshecho supone un valor agregado para esta fibra.

Coco. La fibra de coco es barata y está disponible en muchos países tropicales y semitropicales y se obtiene como residuo de las fibras de los frutos del cocotero, del grueso mesocarpio o cáscara del coco. Esta fibra es totalmente biodegradable y de entre 0,18-0,36 mm de diámetro. Ha sido ampliamente utilizada como material de refuerzo en cementos y en techos debido a su mayor resistencia frente a otras fibras naturales $(9,10)$. También como sustrato artificial en agricultura, pudiendo ser incorporada al suelo como compost tras su uso (11).

Denim. Se dispone de una fuente enorme de materia prima y sería interesante definir un producto comercial que permitiera reintroducir este producto en el mercado $(12,13)$. Los pantalones denim son un ejemplo de ropa que se deshecha. La eliminación final de estos desechos textiles está causando contaminación del suelo y del agua principalmente a través del teñido, proceso que utiliza un alto contenido de sustancias como azufre, naftol, jabones, enzimas y colorantes (12). Para la fabricación de las muestras se obtuvieron fibras de algodón de residuos denim etiquetado por el fabricante como $100 \%$ algodón cortado en piezas cuadradas que fue deshilachado utilizando un molino de cuchillas. Se trata de fibras de algodón de entre 0,01 y 0,03 mm de diámetro.

La principal aplicación de la fibra de algodón es la elaboración de ropa. La función de la ropa es proteger el cuerpo humano del frío y del calor por lo que el estudio de estas fibras debe basarse en esta premisa (14). Los residuos textiles pueden proceder de la basura de la industria textil o pueden ser el resultado de prendas que ya no se usan. En la Unión Europea se generan más de 6 millones de toneladas de prendas textiles descartadas por los consumidores de las que solo un $25 \%$ son recicladas por organizaciones benéficas. El resto se destina a vertederos o son incineradas. En este proceso se libera una cantidad sustancial de gases de efecto invernadero como resultado de la descomposición de la celulosa. Existe, 
por tanto la necesidad de un medio eficiente de reciclaje de residuos de algodón.

\subsection{Muestras de no tejidos}

A partir de las fibras descritas se han elaborado muestras de no tejido unidas de manera mecánica mediante una punzonadora. La punzonadora permite la fabricación de no tejidos sin utilizar ningún tipo de ligante. En el proceso de fabricación las fibras pasan a través de un mecanismo de agujas que se introducen en medio de las fibras y gracias al movimiento alternado de las agujas que poseen pequeños ganchos salientes, las fibras se entrelazan entre sí (6). Una de las ventajas de la fabricación no tejida es que generalmente en un proceso continuo directamente desde la materia prima.
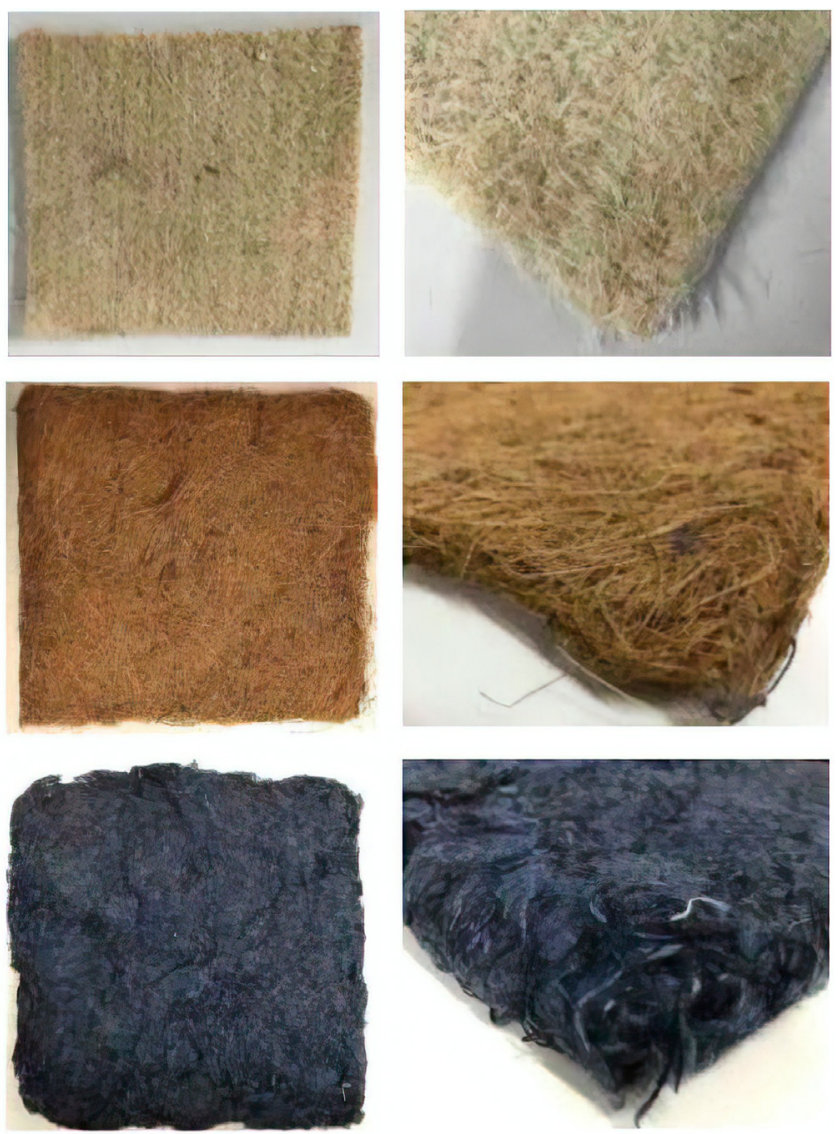

Figura 1. Fotografías de las muestras fabricadas, de arriba abajo: de fibras de fique, de fibras de coco y de fibras de algodón reciclado de tejido denim.

En la Figura 1 se muestran fotos de los no tejidos fabricados. Las muestras tienen dimensiones de $25 \mathrm{~cm}$ x $25 \mathrm{~cm}, 2,5 \mathrm{~cm}$ de espesor y $80 \mathrm{~kg} / \mathrm{m}^{3}$ de densidad aproximada. Estudios previos llevados a cabo con no tejidos de fique indicaron esta densidad como apropiada por la relación entre la cantidad de fibras empleada y las prestaciones acústicas y térmicas del material diseñado (15).

\subsection{Técnicas de caracterización}

\subsubsection{Conductividad térmica}

La conductividad térmica $\lambda$ de las muestras fabricadas se midió por medio de un medidor de flujo de calor mode- lo HFM 436 Lambda de Neszcht. El método basado en el medidor de flujo de calor o la técnica de placa caliente guardada es una técnica de ensayo normalizado (16). La muestra se coloca entre dos placas calentadas a diferentes temperaturas. El flujo de calor $q$ a través de la muestra se mide mediante un transductor calibrado de flujo de calor. La medida se realiza una vez que se ha alcanzado el equilibrio térmico. Las temperaturas de las placas están controlados por sistemas bidireccionales de calentamiento/ enfriamiento Peltier integrados en un intercambiador de calor de aire forzado que genera un flujo de bucle cerrado.

\subsubsection{Rigidez dinámica}

Las medidas de rigidez dinámica se realizaron de acuerdo con la norma UNE EN 29052-1 (17) que describe el procedimiento para la determinación de la rigidez dinámica para materiales con superficies lisas. El procedimiento de medida está basado en la medida de la frecuencia de resonancia de un sistema masa-muelle donde el muelle es el material objeto de estudio y la masa una placa de acero cuadrada de dimensiones 200 x $200 \mathrm{~mm}^{2}$ y unos $8 \mathrm{~kg}$ de peso. Durante los ensayos la muestra se colocó entre una superficie lisa y rígida (suelo del laboratorio) y las placas de acero. Las superficies entre las que se coloca la muestra deben ser lo suficientemente rígidas para evitar ondas de flexión en el intervalo de frecuencias de interés. Conocida la frecuencia de resonancia del sistema, la rigidez dinámica aparente por unidad de superficie de la muestra se calcula mediante la expresión:

$$
s_{t}^{\prime}=4 \pi^{2} m_{t}^{\prime} f_{r}^{2}
$$

donde $m_{t}$ es la masa total por unidad de superficie del sistema ensayado en $\mathrm{kg} / \mathrm{m}^{2}$ y $f_{r}$ es la frecuencia de resonancia. La frecuencia de resonancia de la vibración vertical del sistema muestra y placa de carga se determinó utilizando como excitación una señal de ruido blanco aplicada a la muestra mediante un excitador Modelo 4824 de Brüel\&Kjaer.

\subsubsection{Coeficiente de absorción sonora}

Para la medición del coeficiente de absorción a se ha utilizado una pistola de impedancia. Esta técnica, comercializada por Microflown Thecnologies consiste en un altavoz esférico de $11 \mathrm{~cm}$ de diámetro y una sonda PU (presión-velocidad) (18-20). La distancia entre el altavoz y la sonda PU se mantiene constante e igual a $27 \mathrm{~cm}$. El altavoz genera el sonido y la sonda PU mide la presión sonora y la velocidad de las partículas del aire sobre la superficie del material. La relación entre la presión $\mathrm{P}$ y la velocidad $\mathrm{U}$ de la partícula permite calcular el coeficiente de reflexión y por lo tanto, el coeficiente de absorción. Un software lleva a cabo el control de la medición y realiza todos los cálculos necesarios.

La pistola de impedancia permite caracterizar de forma fácil y rápida el coeficiente de absorción de un material independientemente de su ubicación siempre que su superficie sea superior a $20 \mathrm{~cm} \mathrm{x} 20 \mathrm{~cm}$ y no requiere de preparación previa de las muestras. Esta técnica ofrece resultados comparables a los de un tubo de impedancia a partir de los 400 $\mathrm{Hz}(20)$. 


\section{RESULTADOS Y DISCUSIÓN}

\subsection{Propiedades térmicas}

Como ya se ha indicado una de las medidas para disminuir el consumo energético de un edificio es dotarlo de un aislamiento térmico adecuado. Desde el punto de vista térmico el CTE en su DB-HE clasifica los productos mediante su conductividad térmica $\lambda$ y el factor de resistencia a la difusión del vapor de agua $m$. Considera aislante térmico aquel material que tiene una conductividad térmica menor que $0,05 \mathrm{~W} /$ $\mathrm{m} \times \mathrm{K}$ y una resistencia mayor que $0,025 \mathrm{~m}^{2} \times \mathrm{K} / \mathrm{W}$. En general y salvo justificación, los valores de diseño serán los definidos para una temperatura de $10^{\circ} \mathrm{C}$.

En la Tabla I se muestran a $10^{\circ} \mathrm{C}$ los valores medidos para las fibras escogidas y los valores de algunos aislantes térmicos convencionales. Los valores presentados son el promedio de las mediciones realizadas con tres muestras diferentes de iguales características para cada tipo de fibra. En un material fibroso la conductividad térmica es una combinación de la conductividad de la fibra y el aire. El diámetro y morfología de los espacios vacíos que se generan es otro factor importante que fija el valor final de $\lambda$. Esto podría explicar la menor conductividad térmica de las muestras fabricadas a partir de las fibras de denim (ver Tabla I). Dado el menor diámetro que presentan y su mayor flexibilidad, la mayor concentración de fibras permitiría generar cavidades de menor diámetro que ofrecerían mayor resistencia térmica (21).

Tabla I. Valores de la conductividad térmica a $10^{\circ} \mathrm{C}$ de los tres tipos de fibras y de algunos materiales aislantes térmicos convencionales (valores extraídos del CTE).

\begin{tabular}{|l|c|}
\hline Materiales & $\boldsymbol{\lambda}(\mathbf{W} / \mathbf{m} \times \mathbf{K}) \mathbf{a ~} \mathbf{~ o}^{\mathbf{0}} \mathbf{C}$ \\
\hline Fique & 0,039 \\
\hline Coco & 0,041 \\
\hline Denim & 0,038 \\
\hline Fibra de vidrio & 0,040 \\
\hline Lana de roca & 0,038 \\
\hline Espuma de poliuretano & 0,029 \\
\hline EPS & 0,039 \\
\hline Corcho & 0,040 \\
\hline
\end{tabular}

Es importante estudiar el efecto de la temperatura y la humedad sobre el valor de la conductividad térmica. En la Figura 2 se ha representado la conductividad térmica $\lambda$ medida en función de la temperatura para valores entre 5 y $50^{\circ} \mathrm{C}$. En el rango de temperaturas analizado el valor de $\lambda$ aumenta ligeramente con la temperatura en todos los casos. Se han llevado a cabo algunos estudios sobre la influencia de la temperatura en el valor de la conductividad térmica para materiales como una lana mineral o una espuma de poliuretano (22, 23). Los resultados mostraron que en general en los materiales fibrosos cuanto mayor es la temperatura y la humedad mayor es el valor de la conductividad térmica. Este hecho es debido a la alta conductividad del agua que es unas 25 veces mayor que la del aire a temperatura ambiente. Aunque no se ha llevado a cabo un estudio sobre el efecto de la humedad un inconveniente de las fibras naturales es que son hidrófilas. La cantidad de absorción de agua depende del valor de $m$ del material, se asume que la absorción de agua de un material es mayor cuanto menor es el valor de $m$. Para las fibras naturales, los valores de la resistencia al vapor oscilan entre 1 y 2 , la de la espuma de poliuretano oscila entre 60 y 150.

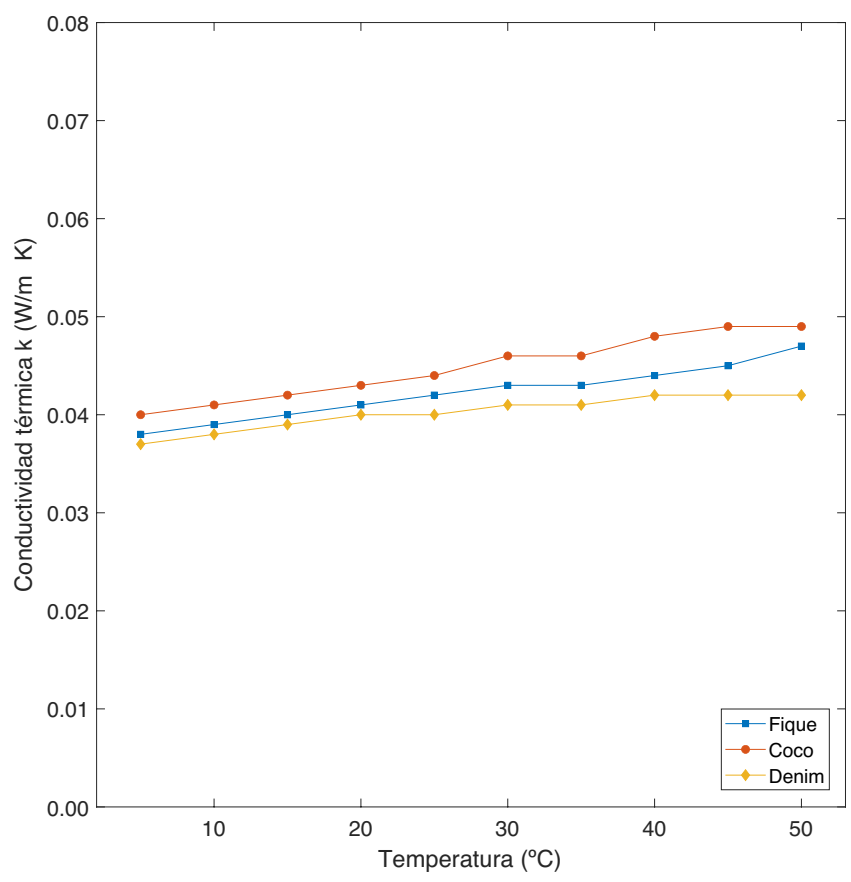

Figura 2. Conductividad térmica $\lambda$ en función de la temperatura entre 5 y $50^{\circ} \mathrm{C}$ para los materiales fabricados

Cuando se analiza una vivienda energéticamente, el espesor del aislamiento térmico es una de las cuestiones básicas. El espesor óptimo del aislante debe tener en cuenta, entre otros, el tipo de edificio, las condiciones climáticas, los materiales de construcción, con el objetivo de reducir el consumo anual de energía para calentar o enfriar un edificio (24). Basado en los valores de $\lambda$ los materiales fabricados se podrían usar como aislantes térmicos en cerramientos exteriores, particiones interiores y cubiertas. En la Tabla II se ha estimado la transmitancia térmica $U$ que resultaría al usar los materiales fabricados como aislantes térmicos para dos espesores, 5 y $10 \mathrm{~cm}$, en cerramientos combinados con un ladrillo perforado de medio pie o con un tablero de partículas de madera. La normativa se encuentra en constante cambio y los espesores del aislamiento térmico de los edificios han aumentado su valor. El incremento de los espesores reconoce al aislamiento como el elemento fundamental sobre el que diseñar cualquier política de ahorro de energía en los edificios y para cumplir con los compromisos derivados de las diferentes directivas europeas. Para evitar descompensaciones en el aislamiento entre las diferentes partes del edificio el CTE en su DB-HE incluye tablas de requerimientos mínimos en los que se indica el aislamiento mínimo que deben tener los elementos que configuran la envolvente del edificio, las medianerías y las particiones interiores. El requerimiento viene determinado por la zona climática. Para el espesor de $10 \mathrm{~cm}$ se cumplen los requisitos térmicos exigidos por el Código Técnico de la Edificación para muros y suelos pertenecientes a la envolvente térmica (Tabla 3.1.1.a-HE1) y para delimitaciones de particiones interiores de igual y distintos usos en todas las zonas climáticas (Tabla 3.2-HE1). Para cumplir los requisitos mínimos exigidos para delimitaciones de particiones interiores de igual y distintos usos es suficiente con un espesor de $5 \mathrm{~cm}$ para el aislante térmico. 
Tabla II. Valores de la transmitancia térmica $U$ que resultan de combinar cada uno de los materiales fabricados con un ladrillo perforado de medio pie y con tableros de partículas de madera. En el cálculo se han considerado dos espesores diferentes, 5 y 10 $\mathrm{cm}$, para el aislante térmico fabricado con las fibras naturales.

\begin{tabular}{|c|c|c|c|}
\hline Fibra & $\begin{array}{l}U(10 \mathrm{~cm}) \\
\left(\mathrm{W} / \mathrm{m}^{2} \cdot \mathrm{K}\right)\end{array}$ & $\begin{array}{c}U(5 \mathrm{~cm}) \\
\left(\mathrm{W} / \mathbf{m}^{2} \cdot \mathrm{K}\right)\end{array}$ & Secciones \\
\hline Fique & 0,37 & 0,70 & \\
\hline Coco & 0,39 & 0,74 & \\
\hline Denim & 0,36 & 0,68 & \\
\hline Fique & 0,36 & 0,67 & \\
\hline Coco & 0,38 & 0,71 & \\
\hline Denim & 0,35 & 0,65 & \\
\hline Fique & 0,35 & 0,63 & \\
\hline Coco & 0,37 & 0,67 & \\
\hline Denim & 0,34 & 0,62 & \\
\hline
\end{tabular}

\subsection{Propiedades acústicas}

Desde el punto de vista acústico se discuten las posibilidades de uso de los materiales fabricados para aislamiento acústico y para acondicionamiento acústico.

Aislamiento acústico. Para materiales fibrosos de baja resistividad al caudal del aire, la norma (17) indica el valor de $s^{\prime}{ }_{t}$ medido se puede aproximar a la rigidez dinámica por unidad de superficie $s^{\prime}$. A partir de $s^{\prime}$ se puede estimar la mejora que introducirían los materiales fabricados en el aislamiento acústico de un paramento horizontal o vertical. En la Tabla III se indican los valores medidos para la rigidez dinámica para los tres tipos de muestras. Los valores de la tabla, al igual que para $\lambda$, son el promedio de los valores medidos para tres muestras diferentes para cada tipo de fibra. A efectos comparativos, en la tabla se incluyen también los parámetros acústicos medidos para una lana de roca de espesor y densidad similar al de las muestras fabricadas.

Para describir el aislamiento a ruido aéreo de los elementos constructivos se utiliza el índice global de reducción sonora $R_{W}$, y $R_{A}$ si es ponderado A. El CTE impone valores mínimos para estos parámetros. Para el caso de elementos que proporcionan aislamiento a ruido de impactos además debe conocerse el nivel global de aislamiento a ruido de impactos normalizado, $L_{W}$. Aunque los valores de estos parámetros acústicos deberían obtenerse a partir de mediciones en laboratorio, también se pueden obtener utilizando cálculos teóricos o estimaciones empíricas. No obstante, es importante siempre indicar cuales son las fuentes de los datos utilizados. En este este caso se ha calculado la mejora del índice global de reducción acústica a ruido aéreo $\Delta R_{W}$ en un cerramiento trasdosado en el que la cámara se rellena con estos materiales. Para un elemento de separación horizontal, se ha calculado la reducción del nivel glo- bal de aislamiento acústico a ruido de impactos $\Delta L_{W}$ debida a un suelo flotante en el que la capa elástica estuviera constituida por los materiales fabricados. Estos índices de mejora representan la diferencia entre el índice de reducción acústica, $R_{W}$ o $L_{W}$, del elemento estructural básico con la capa adicional y el elemento estructural básico sin esa capa. El cálculo de la mejora depende del tipo de elemento estructural al que se apliquen estos recubrimientos.

Tabla III. Parámetros acústicos (rigidez dinámica, frecuencia de resonancia $f_{o}$ y mejora al aislamiento a ruido aéreo $\Delta R_{W}$ ) medidos para los tres tipos de muestras fabricadas y para una lana de roca de similar densidad y espesor.

\begin{tabular}{|l|c|c|c|}
\hline Material & $\begin{array}{c}\text { Rigidez } \\
\text { Dinámica } \\
\left(\mathbf{M N} / \mathbf{m}^{3}\right)\end{array}$ & $\begin{array}{c}\text { Frecuencia } \\
\text { resonancia } \\
\boldsymbol{f}_{\boldsymbol{o}}\end{array}$ & $\begin{array}{c}\boldsymbol{\Delta R}_{\boldsymbol{W}} \\
(\mathbf{H z})\end{array}$ \\
\hline Fique & 5 & 98 & 12,1 \\
\hline Coco & 4 & 93 & 12,5 \\
\hline Denim & 6 & 105 & 11,5 \\
\hline Lana de roca & 6 & 106 & 11,4 \\
\hline
\end{tabular}

Para elementos estructurales básicos con un índice de reducción sonora en el rango de 20 a $60 \mathrm{~dB}, \Delta R_{W}$ se puede estimar a partir del cálculo de la frecuencia de resonancia del sistema (25). Se ha considerado un cerramiento constituido por un ladrillo perforado de medio pie de masa por unidad de superficie $m_{1}=160 \mathrm{~kg} / \mathrm{m}^{2}$ trasdosado con una placa de yeso laminado de espesor $1,5 \mathrm{~cm}$ y de una masa por unidad de superficie de $m_{2}=12 \mathrm{~kg} / \mathrm{m}^{2}$. La frecuencia de resonancia del sistema formado por el ladrillo perforado de medio pie de masa $\mathrm{m}_{1}$, una capa de $2,5 \mathrm{~cm}$ de espesor de coco, fique, denim o lana mineral de rigidez dinámica $s^{\prime}$ y una placa de yeso de masa $\mathrm{m}_{2}$ se ha determinado mediante la siguiente expresión (Anexo D UNE EN ISO 12354-1 (25)):

$$
f_{0}=\frac{1}{2 \pi} \sqrt{s^{\prime}\left(\frac{1}{m_{1}^{\prime}}+\frac{1}{m_{2}^{\prime}}\right)}
$$

Los valores $\operatorname{de} f_{\mathrm{o}}$ se han incluido en la Tabla III. Para frecuencias de resonancia comprendidas entre 30 y $160 \mathrm{~Hz}$ la norma indica que $\Delta R_{W}$ se estima a partir de la siguiente expresión:

[3]

$$
\Delta R_{W}=74,4-20 \lg \left(f_{0}\right)-R_{W} / 2
$$

En la expresión [3], $R_{W}$ representa el aislamiento acústico global del elemento estructural básico, que en este caso es de $45 \mathrm{~dB}$. Los valores de $\Delta R_{W}$ se indican en la Tabla III. La mejora introducida por las capas de las fibras de coco o denim son comparables a la que se obtiene con una lana mineral.

Para impedir la transmisión de ruido de impactos los elementos de separación horizontales suelen contar con un suelo flotante. El material aislante a ruido de impactos en un suelo flotante suelen ser materiales elásticos y flexibles, generalmente poliuretano, características que comparten los materiales fabricados a partir del fique, coco y denim. La reducción del nivel de presión sonora de impactos $\Delta L_{W}$ depende de la masa por unidad de superficie de los suelos flotantes y de la rigidez dinámica $s^{\prime}$ de la capa elástica. La norma UNE EN ISO 12354-2 (26) en su Anexo C indica las expresiones que se han de aplicar para obtener la re- 
ducción del nivel de presión acústica de impactos $\Delta L_{W}$ de suelos flotantes. La mejora depende del elemento estructural básico al que se aplica, para soleras de suelo flotante hechas de mortero/cemento o sulfato de calcio, los valores se pueden calcular haciendo uso de la fórmula:

$$
\Delta L_{W}=\left(13 \lg \left(m^{\prime}\right)\right)-\left(14,2 \lg \left(s^{\prime}\right)\right)+20,8 d B
$$

Para suelos flotantes en asfalto o construcciones de suelo flotante seco, los valores se pueden calcular haciendo uso de la fórmula:

[5] $\quad \Delta L_{W}=\left(\left(-0,21 m^{\prime}\right)-5,45\right) \lg \left(s^{\prime}\right)+\left(0,46 m^{\prime}\right)+23,8 d B$

En las expresiones [4] y [5], $m^{\prime}$ representa la densidad del suelo flotante y $s^{\prime}$ la rigidez dinámica de la capa elástica. Estas expresiones son válidas siempre y cuando la capa elástica cubra toda la superficie del suelo sin ninguna separación o corte. Los valores que resultan de aplicar las expresiones [4] y [5] se han representado para cada fibra en función de $m^{\prime}$ en la Figura 3 y la Figura 4.

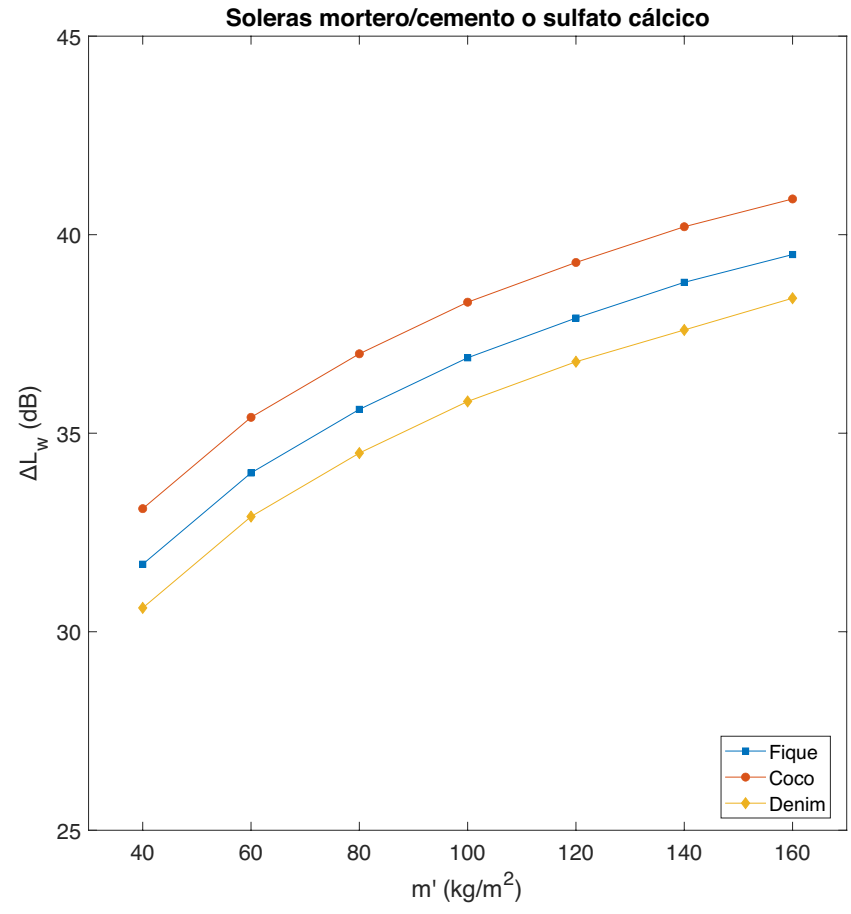

Figura 3. $\Delta L_{W}$ en función de la densidad del suelo flotante para los tres tipos de muestras fabricados en el caso de soleras de mortero.

Dependiendo de la densidad del forjado el Código Técnico exige que el suelo flotante tenga una mejora u otra con respecto a los aislamientos a ruido de impactos y a ruido aéreo. Según el DB-HR para forjados homogéneos aligerados de masa menores, a partir de $175-180 \mathrm{~kg} / \mathrm{m}^{2}$, y en el caso más desfavorable se requieren mejoras en el aislamiento acústico a ruido de impactos por encima de $26 \mathrm{~dB}$, valores que se conseguirían con todos los materiales fabricados (véase Figura 4).

Es importante tener en cuenta que el aislamiento acústico obtenido para un elemento constructivo in situ es, en general, menor que el aislamiento acústico teórico. Esto se debe a que el aislamiento acústico final depende no sólo de los elementos constructivos proyectados para la separación

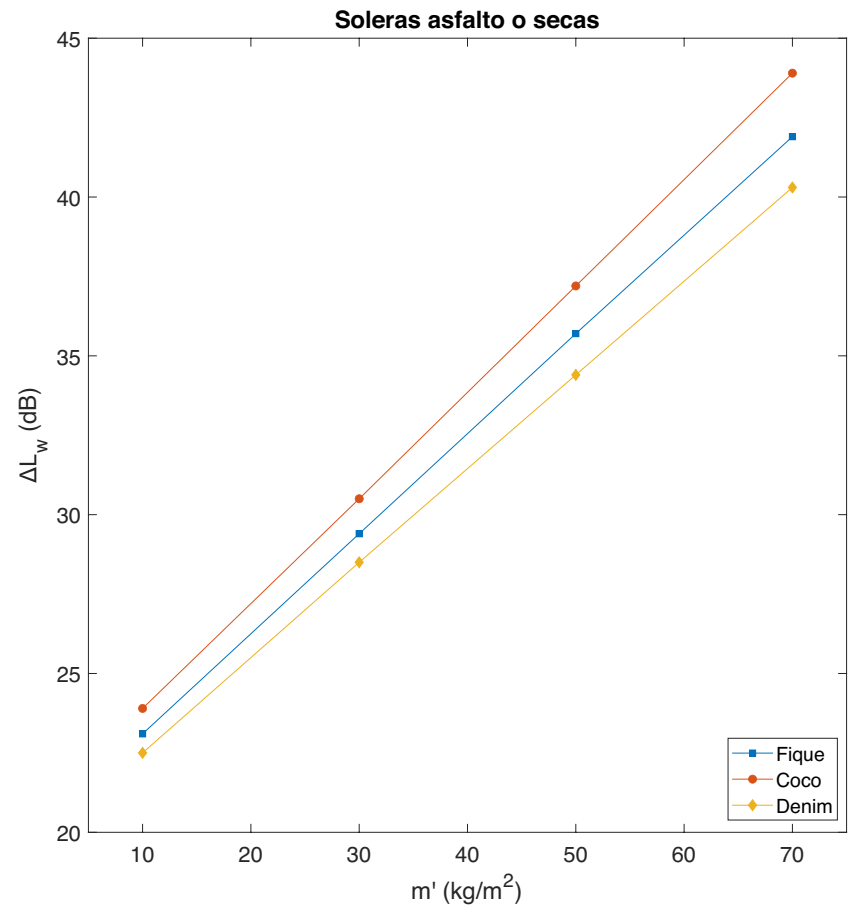

Figura 4. $\Delta L_{W}$ en función de la densidad del suelo flotante para los tres tipos de muestras fabricados en el caso de soleras de asfalto o secas.

sino también de los encuentros entre ellos y de la calidad de su ejecución. El tipo y calidad de la conexión entre elementos fija la transmisión acústica indirecta o por flancos. El CTE indica que aunque esta diferencia puede variar sensiblemente en funcion de los tipos constructivos, de modo orientativo, puede decirse que es superior en $5 \mathrm{dBA}$. Por ejemplo, en un elemento separador de doble hoja, el tipo de material absorbente que se instala en la cámara pierde relevancia frente a la desolidarización de los cerramientos mediante bandas perimetrales. En este tipo de cerramientos o medianeras el camino dominante de la transmisión del sonido es el que conecta la hoja interior con los elementos de separación horizontales. Si este camino no se anula adecuadamente, el resto de materiales que se coloquen en la solución no aportarán ninguna mejora.

Acondicionamiento acústico. En recintos que suelen tener las superficies reflectantes y que son de un tamaño considerable conviene elegir materiales absorbentes con los que recubrir las paredes o el techo, de modo que el tiempo de reverberación se mantenga dentro de unos límites correctos. De esta manera se consigue que disminuyan los niveles de ruido en el interior de los edificios, así como una mayor inteligibilidad de la palabra, importante en recintos como aulas y salas de conferencias.

Los valores numéricos medidos para el coeficiente de absorción $\alpha$ en bandas de tercio de octava se indican en la Tabla IV. Se ha incluido en la tabla el Índice de Reducción Sonora NRC (Noise Reduction Coefficient) calculado como la media aritmética de los valores medidos en las bandas de octava entre 250 y $2000 \mathrm{~Hz}$. En la Figura 5 se han representado gráficamente estos resultados y se han incluido los valores medidos para la lana de roca. Los valores de $\alpha$ indican que las muestras fabricadas son buenos absorbentes acústicos en frecuencias intermedias y altas. Comportamiento comparable al obtenido por otros autores para materiales fabricados 
con otras fibras naturales (27). Ensayos llevados a cabo con la fibra de kenaf sobre la influencia de la humedad indican que no parece que la humedad tenga un efecto significativo sobre el valor del coeficiente de absorción (28). No obstante, un estudio sobre la influencia de la humedad en las muestras fabricadas es muy conveniente.

Tabla IV. Valores del coeficiente del coeficiente de absorción $\alpha$ en bandas de octava medido con la sonda P-U para las tres fibras naturales y la lana de roca de espesor $2,5 \mathrm{~cm}$.

\begin{tabular}{|l|c|c|c|c|}
\hline \multirow{2}{*}{} & \multicolumn{4}{|c|}{ Coeficiente de absorción $\boldsymbol{\alpha}$} \\
\cline { 2 - 5 } & Fique & Coco & Denim & $\begin{array}{c}\text { Lana de } \\
\text { roca }\end{array}$ \\
\hline $\mathbf{1 2 5} \mathbf{~ H z}$ & 0,19 & 0,21 & 0,08 & 0,02 \\
\hline $\mathbf{2 5 0} \mathbf{~ H z}$ & 0,12 & 0,15 & 0,06 & 0,16 \\
\hline $\mathbf{5 0 0} \mathbf{~ H z}$ & 0,29 & 0,31 & 0,26 & 0,30 \\
\hline $\mathbf{1 0 0 0} \mathbf{~ H z}$ & 0,46 & 0,40 & 0,71 & 0,85 \\
\hline $\mathbf{2 0 0 0} \mathbf{~ H z}$ & 0,85 & 0,77 & 0,96 & 0,80 \\
\hline $\mathbf{4 0 0 0} \mathbf{H z}$ & 0,87 & 0,82 & 0,96 & 0,90 \\
\hline NRC & 0,43 & 0,41 & 0,51 & 0,53 \\
\hline
\end{tabular}

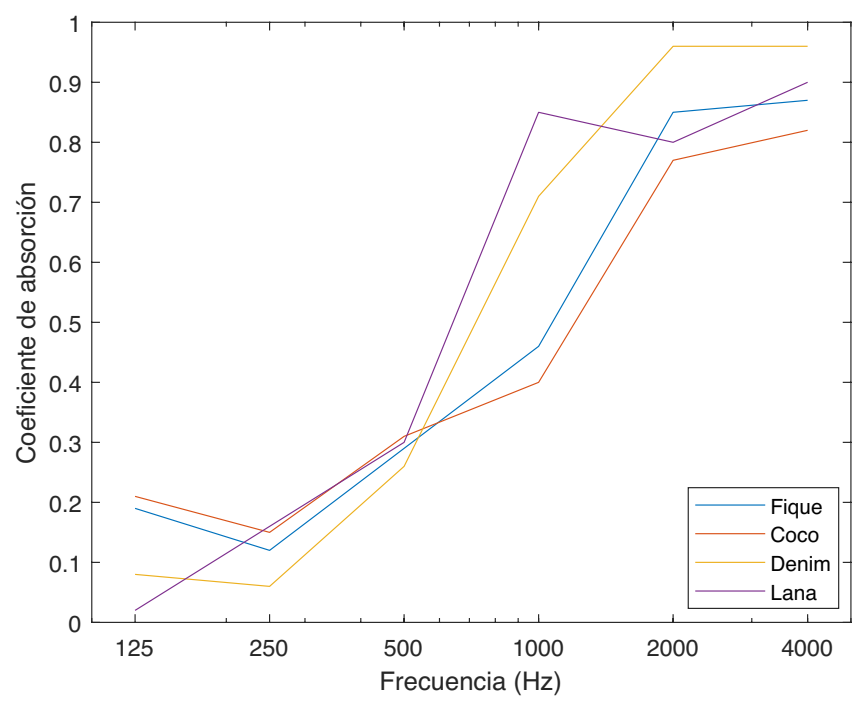

Figura 5. Coeficiente de absorción sonora medido con la sonda PU para los tres tipos de muestras fabricadas. Para efectos comparativos también se ha incluido los valores medidos para la lana de roca.

Durante la selección de un material además de sus propiedades acústicas y térmicas se deben tener en cuenta otros factores como la disponibilidad, el impacto energético o el coste. El factor económico condiciona totalmente la arquitectura y por tanto, la selección de los materiales. Este factor es muy variable en todos los aspectos ya que no sólo depende del material, sino también del fabricante, el transportista, las dimensiones, la ubicación y el tipo de obra que tendrán gran influencia sobre el presupuesto. En cada obra se deberán seleccionar materiales que cumplan con los requerimientos y que se ajusten al presupuesto con el que se cuente, lo que influirá mucho en la selección del material. Se ha realizado una estimación del coste de fabricación de las muestras basados exclusivamente en el coste de mano de obra, en el coste de la materia prima y en la energía eléctrica consumida para su fabricación. Cada panel de 25 x $25 \mathrm{~cm}$ tendría un coste aproximado de $1 €$ de manera que por metro cuadrado el coste sería de aproximadamente $16 €$. En la Tabla V se muestran los costes por metro cuadrado de algunos materiales convencio- nales usados en el sector de la construcción como aislantes térmicos y acústicos.

Tabla V. Coste de aislantes térmicos y acústicos por metro cuadrado.

\begin{tabular}{|l|c|}
\hline Muestras fique, coco y denim & $16 € / \mathrm{m}^{2}$ \\
\hline Poliestireno expandido & $3,2 € / \mathrm{m}^{2}$ \\
\hline Poliestireno extraído & $9,6 € / \mathrm{m}^{2}$ \\
\hline Fibra de vidrio & $3,2 € / \mathrm{m}^{2}$ \\
\hline Lana de roca & $3,6 € / \mathrm{m}^{2}$ \\
\hline Espuma de poliuretano & $11 € / \mathrm{m}^{2}$ \\
\hline
\end{tabular}

No obstante, aunque se puede estimar el coste de producción, solo después de definir y estudiar un posible producto comercial este análisis se puede hacer con propiedad. Es importante considerar todos los factores, beneficios y repercusiones a largo plazo a través de un análisis del ciclo de ida. La evaluación del ciclo de vida (Life Cicle Assesment LCA) implica evaluar los impactos ambientales de un producto, proceso o actividad de manera integral, observando el ciclo de vida completo del producto o del proceso desde la extracción de las materias primas hasta su eliminación (29). Para ello, en el caso de un producto se deberían conocer todas las actividades necesarias para extraer la materia prima incluido el transporte antes del procesamiento, las actividades necesarias para convertir la materia prima y el consumo de energía en el producto deseado, así como el envío del producto al usuario final, la utilización del producto terminado durante su vida útl, una vez que el producto ha cumplido su función prevista y posteriormente se recicla o se devuelve al medio ambiente como residuo. Para modelar el ciclo de vida es importante disponer de toda esta información. Si hacemos un ciclo de vida para los materiales fabricados solo basados en la conductividad térmica el resultado nos saldría muy similar ya que los valores de este parámetro son muy parecidos. Por ello, en el trabajo no se ha incluido un análisis de ciclo de vida.

\section{CONCLUSIONES}

Se han fabricado muestras de no tejidos basados en tres fibras naturales: fique, coco y algodón, estas últimas recicladas a partir de tejido denim. Para su fabricación se ha propuesto el punzonado, una técnica de unión mecánica sin ligante. De estos materiales se ha medido la conductividad térmica, la rigidez dinámica y el coeficiente de absorción sonora. Se han obtenido valores comparables a los que presentan los aislantes térmicos y acústicos usados habitualmente en construcción. También se ha estimado su coste basados en el proceso de fabricación seguido. En resumen, se han presentado las fibras naturales como materia prima y una técnica de fabricación, el punzonado, que pueden constituir una buena alternativa para reducir el consumo de energía y el uso de materiales medioambientalmente nocivos y de esta manera contribuir a la sostenibilidad del edificio. En esta misma línea, uno de los objetivos de la Agenda 2030 para el Desarrollo Sostenible se basa en hacer que los asentamientos humanos sean inclusivos, seguros, resistentes y sostenibles. El uso de materiales locales junto con los recursos renovables son algunos de los puntos clave lo que refuerza el desarrollo y utilización de este tipo de materiales.

No obstante, quedan aspectos comerciales, así como propiedades físicas que se han de tratar y analizar en detalle. Para 
definir un producto comercial propiedades como la resistencia a la compresión, al vapor de agua, el comportamiento frente al fuego... también han de ser estudiados. En este sentido, los materiales desarrollados presentan algunos inconvenientes importantes y que habría que abordar y solucionar para poder extender su uso en construcción. Por ejemplo, su naturaleza hidrófla puede resultar en el debilitamiento de las propiedades de la fibra. Es por ello que, en algunos casos las fibras naturales tienen que ser modificadas y/o tratadas con el fin de dotarlas de propiedades de las que carecen por naturaleza como impermeabilidad, características ignífugas o estabilidad térmica. La resistencia al fuego se obtiene normalmente utilizando aditivos que contienen con frecuencia fósforo, cloro o bromo. También deben ser sometidos a trata- miento de protección contra el ataque de xilófagos, hongos y otras agresiones biológicas.

No obstante, la sostenibilidad es muy importante en la industria de la construcción y crece la preocupación de la sociedad en este sentido. Si los edificios se diseñan y operan adecuadamente, se pueden lograr ahorros significativos de energía. Los materiales de aislamiento térmico tienen un papel importante y son el primer paso lógico para reducir la energía requerida para mantener una buena temperatura interior logrando así, una mejor eficiencia energética. También es importante subrayar de nuevo que poco importa el material aislante elegido, si el método y la calidad de la construcción no son los adecuados ya que éstos últimos fijarán el rendimiento del producto terminado.

\section{REFERENCIAS / REFERENCES}

(1) John, M. J., y Thomas, S. (2008). Biofibres and biocompsites. Carbohydrate polymers, 71(3), 343-364.

(2) Thakur, V. K. (2013). Green composites from natural resources. CRC Press.

(3) Methacanon, P.,Weerawatsophon, U., Sumransin, N., Prahsarn, C., y Bergado, D. T. (2010). Properties and potential applica tion of the selected natural fibers as limited life geotextiles, Carbohydrate Polymers, 82(4), 1090-1096. DOI: 10.1016/j.carbpol.2010.06.036

(4) Summercales, J. Dissanayake, N.P., Virk, A. S., y Hall, W. (2010). A review of bast fibres and their composites. Part 1-Fibres as reinforceents. Composites Part A: Applied Science and Manufacturing, 41 (10), 1329-1335. https://doi. org/10.1016/j.compositesa.2010.06.001

(5) Yousif, B. F., y El-Tayeb, N. S.M. (2009). Mechanical and wear properties of oil palm and glass fibres reinforced polyester composites. International Journal of Precision Technology, 1(2), 213-222. https://doi.org/10.1504/IJPTECH.2009.02638

(6) Navacerrada, M. A., Díaz, C., Pedrero, A., Isaza, M., y Fernández, P. (2016). Caracterización acústica y térmica de no tejidos basados en fibras naturales. Proceedings de EuroRegio 2016, Junio 13-15, Oporto (Portugal)

(7) Delvasto, S., Toro, E., Perdomo, F., y Mejía, R. (2010) An appropiate vacum technology for manufacture of corrugated fique fiber reinforced cementitious sheets. Construction and Building Materials, 24, 187-192. DOI: 10.1016/j.conbuildmat.2009.01.010

(8) Gañán, P., y Mondragón, I. (2002). Surface modification of fique fibers: effects on their physicomechanical properties. Polymer Composites 23, 383-394. https://doi.org/10.1002/pc.10440

(9) Hosseini Fouladi, M., Ayub, M. y Jailani Mohd, M. (2011). Analysis of coir fiber acoustical characteristics. Applied Acoustics 72, no. 1, pp. 35-42, 2011. DOI: 10.1016/j.apacoust.2010.09.007

(10) Mathura, N. y Cree, D. (2016). Characterization and mechanical property of Trinidad coir fibers," Journal of Applied Polymer Science, vol. 133, no. 29, pp. 1-9, 2016. https://doi.org/10.1002/app.43692

(11) Hwang, Ch-L.,Tran, V., Hong J., y Hsieh, Y. (2016). Effects of short coconut fiber on the mechanical properties, plastic cracking behavior, and impact resistance of cementitious composites. Construction and Building Materials 127, $984-992$. DOI: 10.1016/j.conbuildmat.2016.09.118

(12) Peña-Pichardo, P., Martínez-Barrera, G., Martínez-López, M., y Ureña-Núñez, F. (2018). Recovery of cotton fibers from waste blue-jeans and its use in polyester concrete. Construction and Building Materials 177, 409-416. DOI: 10.1016/j. conbuildmat.2018.05.137

(13) Wangecheng, L., Shuyan, L., Tian, L., Tuan, L., Jinwen, Z., y Hang, L.(2019). Eco-friendly post-consumer cotton waste recycling for regenerated cellulose fibers. Carbohydrate Polymers 206, 141-148. DOI: 10.1016/j.carbpol.2018.10.046

(14) Briga-Sa, A., Nascimiento, D., Teixeira, N., Pinto, J., Caldeira, Varum, H., y Paiva, A. (2013). Textile waste as an alternative thermal insulation building material solution. Construction and Building Materials 38, 155-160. https://doi. org/10.1016/j.conbuildmat.2012.08.037

(15) Navacerrada, M. A., Díaz, C.; y Fernández P. (2014). Characterization of a material based on short natural fique fibers. Bioresources 9(2), 3480-3496. DOI: 10.15376/biores.9.2.3480-3496

(16) UNE -EN ISO 12667: 2002. Materiales de construcción. Determinación de la resistencia térmica por el método de la placa caliente guardada y el método del medidor de flujo de calor. Productos de alta y media resistencia térmica.

(17) UNE-EN 29052-1:1994. Acústica. Determinación de la rigidez dinámica. Parte 1: materiales utilizados bajo suelos flotantes de viviendas.

(18) Tijs., E. (2013). Study and development of an in situ acoustic absorption measurement method. PhD, University of Twente ( The Netherlands).

(19) Lanoye R., de Bree, H. E., Lauriks, W., y Vermeir, G. (2004). A practical device to determine the reflection coefficient of acoustic materials in-situ based on a Microflown and micropone sensor. Proceedings of ISMA. Leuven.

(20) Pedrero, A., Navacerrada, M. A., de la Prida, D., Iglesias, L., y Díaz-Chyla, A. (2020). On the accuracy of the sound absorption measurment with an impedance gun. Applied Acoustics 158, 107039. DOI: 10.1016/j.apacoust.2019.107039

(21) Navacerrada, M. A., Fernández, P., Díaz, C., y Pedrero, A. (2013). Thermal and acoustic properties of aluminium foams manufactured by the infiltration process. Applied Acoustics 74, 496-501. https://doi.org/10.1016/j.apacoust.2012.10.006 
(22) Abdou, A.A., y Budaiwi, I. M. (2005). Comparison of thermal conductivity measurements of building insulation materials under various operating temperatures. Journal of Building Physics 29(2), 171-184. https://doi. org/10.1177/1744259105056291

(23) Karamanos, A., Hadiarakou, S., y Papadopoulos, A. M. (2008). The impact of temperature and moisture on the thermal performance of stone wool. Energy Building 40, 1402-1411.

(24) Kaynakli, O. (2012). A review of the economical and optimum thermal insulation thickness for building applications, Renewable and Sustainable Energy Reviews 16, 415-425. https://doi.org/10.1016/j.rser.2011.08.006

(25) UNE EN ISO 12354-1: 2018. Acústica de edificios. Estimación del rendimiento acústico de los edificios a partir del rendimiento de los elementos. Parte 1: Aislamiento acústico a ruido aéreo entre recintos.

(26) UNE EN ISO 12354-2: 2018. Acústica de edificios. Estimación del rendimiento acústico de los edificios a partir del rendimiento de los elementos. Parte 2: Aislamiento acústico a ruido de impactos entre recintos.

(27) Berardi, U., y Iannace, G. (2015). Acoustic characterization of natural fibers for sound absorption applications. Building and Environment 94, 840-852. DOI: 10.1016/j.buildenv.2015.05.029

(28) D’Alessandro, F., Baldinelli, G., Bianchi, F., Sambuco, S., y Rufini, A. (2018). Experimental assessment of the water content influence on thermo-acoustic performance of building insulation materials. Construction and Building Materials 158, 264-274. https://doi.org/10.1016/j.conbuildmat.2017.10.028

(29) Pargana, N., Pinheiro, M. D., Silvestre, J. D., de Brito, J. (2014). Comparative environmental life cycle assessment of thermal-insulation materials of buildings. Energy and Buildings 82, 466-481. https://doi.org/10.1016/j.conbuildmat.2017.10.028 